Instituto Internacional de Investigación y Desarrollo Tecnológico Educativo INDTEC, C.A.

DOI: https://doi.org/10.29394/scientific.issn.2542-2987.2017.2.6.6.113-133

OAI-PMH: http://www.indteca.com/ojs/index.php/Revista Scientific/oai

\title{
Acciones Ambientalistas en la Recolección de Basura Doméstica
}

Autor: Eudis Ramón Osorio García Universidad Pedagógica Experimental Libertador, UPEL eudiseineidis@gmail.com

Barinas, Venezuela

\begin{abstract}
Resumen
El presente artículo es producto de un estudio orientado con la finalidad de Realizar Acciones Ambientalistas en la Recolección de Basura Doméstica, dirigidas a los Habitantes del Sector José Gregorio Hernández, parroquia Ciudad Bolivia, municipio Pedraza, estado Barinas. El objetivo general del estudio es Realizar acciones ambientalistas en la recolección de basura doméstica, dirigidas a los habitantes del sector José Gregorio Hernández, parroquia Ciudad Bolivia, municipio Pedraza, estado Barinas. Se enmarca dentro de la naturaleza cualitativa, para ello se utilizó la recolección de los testimonios expuesto por los sujetos de estudio. La investigación de acuerdo a su tipo se fundamentó en una metodología de Investigación Acción Participativa (IAP), el diseño de esta fue de campo. Así como se consideró como sujetos de estudios a 65 familias, se tomó el método de muestreo no probabilístico intencional y se seleccionaron como muestra veinte (20) familias, de las cuales se tomaron tres (03) jefes de familia en este sentido, la información requerida para esta investigación fue recolectada a través de la técnica de la observación directa y la entrevista. De igual forma se dio el proceso de triangulación. Culminando que a través del desarrollo de acciones ambientalistas en la recolección de la basura doméstica se logra el manejo adecuado de desechos sólidos en el Sector José Gregorio Hernández, como una acción que permitió el mejoramiento sustancial del ambiente y salud de la comunidad y sus habitantes.
\end{abstract} doméstica.

Palabras clave: acciones ambientalistas; recolección; basura

Fecha de Recepción: 11-04-2017 Fecha de Aceptación: 28-06-2017 


\title{
Environmental Actions in the Collection of Domestic Garbage
}

\begin{abstract}
This article is the result of a study aimed at Performing Environmental Actions in the Collection of Domestic Waste, addressed to the Inhabitants of the José Gregorio Hernández Sector, Ciudad Bolivia parish, Pedraza municipality, Barinas state. The general objective of the study is to carry out environmental actions in the collection of domestic waste, directed to the inhabitants of the sector José Gregorio Hernández, parish Ciudad Bolivia, Pedraza municipality, Barinas state. It is framed within the qualitative nature, for it was used the collection of the testimonies exposed by the subjects of study. The research according to its type was based on a methodology of Participatory Action Research (IAP), the design of this was field. Just as 65 families were considered as subjects of study, the method of intentional nonprobabilistic sampling was taken and twenty (20) families were selected as sample, of which three (03) heads of household were taken in this sense, the information Required for this research was collected through the technique of direct observation and interview. The triangulation process was similar. Culminating that through the development of environmental actions in the collection of domestic waste is managed the proper management of solid waste in the Sector Jose Gregorio Hernandez, as an action that allowed the substantial improvement of the environment and health of the community and its inhabitants.
\end{abstract}

Keywords: environmental actions; collection; domestic waste. 


\section{Introducción}

Durante muchos años, el hombre, a través de sus prácticas diarias de tipo doméstico, comercial, industrial; requiere de procesos sencillos 0 complejos que generan una diversidad de productos e igualmente de desechos que consideran como inservibles, pero que tienen utilidad; a estos se les denomina: residuos. Dentro de estos, se encuentran diferentes tipos; clasificados de acuerdo con su estado (líquido, sólido, gaseoso), a su origen (residencial, comercial, industrial, entre otras), a su manejo (peligroso e inerte) y por último a su composición (orgánica e inorgánica).

Lo antes mencionado, permite decir que los residuos pueden ser aprovechadas siempre y cuando desde el momento inicial de sus uso o desusos del recipiente se oriente el para qué y cómo sirven para formar, organizar, reutilizar, todo aquello que en un momento dentro del hogar empresa, escuela, organismos e instituciones, puede ser utilizados nuevamente, como una manera de evitar la contaminación por ellos. Es por eso que, cada día que pasa, la producción de residuos va creciendo exageradamente, originando una problemática ambiental como la contaminación a recursos naturales y la contaminación visual entre otros. De acuerdo con lo antes expuesto Annecca, citado en Padilla (2017), afirma lo siguiente:

En países industrializados como Estados Unidos, Japón, Rusia, teniendo sólo la cuarta parte de la población mundial, utilizan el $80 \%$ de los recursos naturales del planeta en las exigencias propias del desarrollo tecnológico que se lleva a cabo en estos países. Estos modelos de desarrollo exigen grandes cantidades de recursos y energía para transformar la materia, pero generalmente la producción de bienes utilizables va acompañada de una gran cantidad de desechos no deseables que contaminan al ambiente. De igual forma los estilos de vida se distinguen por el consumo indebido de productos y envases desechables, haciendo que la cultura del 
desperdicio se generalice bajo el auspicio de frases tales como "Úselo y tírelo", provocando de esta manera, desmesurada cantidades de residuos contaminantes (pág. 2).

Es decir, que muy a pesar de los procedimientos que se ponen en práctica para la transformación de desechos domésticos, no son suficientes para satisfacer la demanda por la gran cantidad de desechos que emergen de los hogares, de acuerdo a lo expuesto por el autor aún y cuando en los países desarrollados utilizan grandes tecnologías para ello se hacen insuficientes, sin embargo, en Venezuela por lo que se puede observar no se cuenta con tales procedimientos y por ende los focos de contaminación son evidentes.

Es decir, que el venezolano no analiza su conducta en lo que respecta con el estado ambiental, por lo que se deduce que sólo destaca importante los problemas que afectan su entorno inmediato, como es el humo y la basura, ya que los mismos son más tangibles para su efecto visual debido que los asocian a un servicio público como es el aseo urbano, sin hacer ninguna actividad adicional en el caso de no contar con tal servicio.

En tal sentido, Adán, citado en Temas Ambientales.com (2017), manifiesta que, "en Venezuela se estima que, en promedio, cada persona produce $850 \mathrm{~g}$ de basura por día. Si se agregan los residuos de comercios, hospitales y servicios, la cantidad aumenta en $25-50 \%$, y alcanza hasta $1,2 \mathrm{~kg}$ por persona/día." (pág. s/n). Por lo antes expuesto, y si se toman en cuenta los datos del Instituto Nacional de Estadística, donde se reflejó una población total de 28.946.101 personas en el Censo de 2011 y se proyectó que para el 2015 la población alcanzaría 30.620.404 personas, sino se toman las medidas adecuadas contra el control de desechos orgánicos e inorgánicos, el territorio nacional prácticamente se inundará de basura.

Es importante resaltar que la parroquia Ciudad Bolivia del municipio Pedraza no está exenta de la grave contaminación ambiental en la cual está 
inmersa la población, esto se debe a la gran cantidad de residuos sólidos que son arrojados al aire libre en un vertedero que aproximadamente cubre $1.5 \mathrm{~km}$ sin tener ninguna medida de seguridad e higiene. En tal sentido, esto es un problema palpable de contaminación de los suelos y al mismo tiempo se presenta la contaminación del aire, principalmente en las comunidades aledañas a los sectores El Banquito, La Esperanza y Vista Hermosa I y II.

Ante los aspectos señalados, se suma que el origen de la problemática radica en que todos los desechos sólidos recolectados en el municipio son depositados en el área de vertedero, no dándole ningún tratamiento, ni mantenimiento con el transcurrir del tiempo se esparcen fuera del lugar establecido para ello, generando focos de contaminación, deterioro de la vialidad y del ecosistema.

Es de acotar, que a pesar de existir el sistema de recolección de basura, el funcionamiento no es el más idóneo, por lo que los pobladores arrojan la basura en los lugares menos adecuados, aunado a ello por algunos sectores de la población el servicio de aseo urbano no realiza la recolección. De igual manera, la contaminación por desechos sólidos alcanza las riberas del río Canagua, donde se puede evidencias que las personas arrojan la basura en sus alrededores y las lluvias arrastran estos desechos hasta el fondo del mismo, causando la contaminación del preciado líquido.

En la actualidad, el Sector José Gregorio Hernández, de la parroquia Ciudad Bolivia del estado Barinas, presenta una situación deplorable por la contaminación a causa de la cantidad de desechos sólidos, puesto que el servicio del aseo urbano no hace su recorrido de manera permanente, tardando incluso hasta dos y tres semanas en pasar, aunado a ello, tiene un solo lugar de la comunidad como centro de recolección, y muchos de los habitantes se les hace más fácil tirar los desechos en la maleza a expensas de los animales, generándose regueros que perjudican toda la comunidad, 
pues los olores putrefactos, generan virus viéndose afectados en su mayoría los niños quienes son los más vulnerables.

Ante la situación de contaminación a causa de los desechos sólidos en la comunidad antes mencionada, los entes municipales manifiestan que no logran realizar la recolección sino una vez por semana por cada sector debido a la falta de transportes recolectores, sin embargo, a veces los hacen cada quince o veintidós días, a esto se suma la poca concienciación de los habitantes. Es por eso, que surgen una serie de interrogantes, en aras de buscar una solución inmediata que responda a los requerimientos y necesidades de la comunidad:

¿En qué lugares depositan los habitantes del Sector José Gregorio Hernández los desechos sólidos?; ¿Cuáles conocimientos tienen dichos habitantes sobre el manejo de desechos sólidos?, ¿Cuáles servicios de recolección de basura se ofrecen en esta comunidad? y ¿Cómo sería el plan de actividades sobre el manejo de residuos o desechos sólidos en la comunidad?

De acuerdo a las interrogantes se generan los objetivos entre ellos el General como es: Realizar acciones ambientalistas en la recolección de basura doméstica, dirigidas a los habitantes del sector José Gregorio Hernández, parroquia Ciudad Bolivia, municipio Pedraza, estado Barinas y entre los específicos: Diagnosticar, en los habitantes del sector José Gregorio Hernández, el conocimiento sobre la recolección de basura doméstica; Estudiar la factibilidad de la propuesta de las acciones ambientalistas en la recolección de la basura doméstica; Diseñar propuesta de las acciones ambientalistas en la recolección de la basura doméstica; Desarrollar acciones sobre el manejo de la recolección de la basura doméstica en la comunidad y Evaluar la propuesta y los resultados de las acciones ambientalistas en la recolección de la basura doméstica. 


\section{Marco Referencial}

De acuerdo con Blanco (2012), en su investigación presenta como propósito determinar los procedimientos utilizados por los habitantes de la comunidad El Samán de Urama en relación con el manejo de los desechos sólidos orgánicos e inorgánicos, referido a recolección, almacenamiento, transporte, tratamiento y disposición final. Desde el punto de vista metodológico corresponde a un estudio descriptivo transeccional contemporáneo; la población seleccionada estuvo conformada por sesenta y cinco (65) familias residentes en dicha comunidad, de los cuales a través del método de muestreo no probabilístico intencional se seleccionaron como muestra veinte (20) familias.

La información fue recolectada a través de dos instrumentos, el primero, según la modalidad de una lista de observación directa contentiva de quince (15) ítems, referidos al almacenamiento, transporte, tratamiento y disposición final de los desechos sólidos orgánicos e inorgánicos, el segundo, conformado por un cuestionario de doce (12) ítems, con alternativas de respuestas dicotómicas (sí - no), referidas a la fase de recolección de los desechos sólidos orgánicos e inorgánicos. Los resultados determinaron con relación a la recolección de los desechos el 70\% utiliza baldes sin tapa, un $20 \%$ bolsas plásticas y un $20 \%$ recipientes plásticos sin tapa; en cuanto a la frecuencia de recolección el 100\% la realizan una vez al día.

En lo concerniente al transporte o dispositivo, utilizado para movilizar los desechos sólidos desde el área de generación hasta el lugar de disposición o almacenamiento final, el $95 \%$ utiliza envases plásticos y el $5 \%$ carretillas. En relación con el almacenamiento el $100 \%$ de los desechos es colocado en un recinto abierto al aire libre; en lo referido a disposición final en su indicador tratamiento el $85 \%$ queman los desechos sólidos en sus propias viviendas y un $45 \%$ la entierran; mientras que el $90 \%$ trasladan los desechos como destino 
final a un lugar cercano a la comunidad y un $5 \%$ lo reciclan por medio de composteros.

\subsection{Concepción de la Educación Ambiental.}

Una de las maneras de evitar el uso irracional de los recursos naturales, que causa degradación en el ambiente, es a través de una educación ambiental, cuya concepción se, adecue a los procesos económicos de una nación. Según Caballero y Col (2006), la educación ambiental en sus inicios tenía un carácter netamente conservacionista; su objeto era la educación y la toma de conciencia sobre la importancia de los recursos naturales, sin considerar los elementos sociales, técnicos y culturales.

Su concepción era la "no explotación de la naturaleza" por parte del hombre, opuesto al modo de producción adaptado por las sociedades para realizar sus actividades económicas, que les permite aprovechar los recursos materiales, para el logro de sus objetivos de subsistencia, o con fines económicos; olvidando lo cultural, lo social y los verdaderos valores morales, espirituales y ambientales de la sociedad.

El concepto de educación ambiental ha sido estudiado desde varias tradiciones intelectuales, por su parte en el Congreso Internacional de Educación y Formación sobre Ambiente, Moscú, citado por Martínez (2012), en donde se considera que la educación ambiental "es un proceso permanente en el cual los individuos y las comunidades adquieren consciencia de su ambiente, aprenden los conocimientos, los valores, las destrezas, la experiencia y también la determinación que les capacite para actuar, individual y colectivamente... (pág. 73). Entre tanto, la educación ambiental, en palabras de Martínez (2007), quien concibe:

La educación ambiental debe plantearse como un conocimiento integrado del medio socio-natural, donde la mente es un sistema activo-participativo, donde todo está en 
relación con todo, como un proceso abierto, flexible y creativo, para la solución de problemas socioambientales... (pág. 72).

Es por eso, que se considera que la educación ambiental es un proceso de reconocer valores y escalar conceptos para crear habilidades y actitudes necesarias que sirven para comprender; así como para apreciar la relación mutua entre el hombre, su cultura o el medio físico circundante. Igualmente González (2003), señala que la educación ambiental significa "Educar en el ambiente, sobre el ambiente y para el ambiente, brindando un conjunto de conocimientos, habilidades, actitudes, valores, disposiciones que ayudaran a los individuos a asumir roles participando activamente con una sensibilidad y responsabilidad solidaria" (pág. 59), ante esto, la educación ambiental propone construir una ciudadanía ambiental capaz de comprender y valorar su medio biofísico y sus problemas asociados, con conciencia de cómo ayudar a resolver esos problemas y motivada a trabajar hacia su solución.

\subsection{Reciclaje: Las $3 R^{\prime} \mathbf{s}$}

\subsubsection{Reduce.}

El desecho que tiene menor impacto ambiental jes el que no se genera! Procura generar la menor cantidad posible de desechos sólidos. Estas son algunas ideas de cómo REDUCIR la cantidad de desechos sólidos en casa, tal como los señala González (2003), cuando dice: Evita utilizar bolsas plásticas, lleva tus propias bolsas al mercado y al supermercado, rechaza las bolsas de plástico. Procura comprar productos con envases retornables y utilízalos. Evita utilizar platos desechables, prefiere los plásticos. Compra productos con la menor cantidad de envolturas. Investiga sobre que empresas son socialmente responsables y procura comprar sus productos. Evita utilizar duroport, este material no puede reciclarse y es altamente contaminante. Utiliza productos concentrados, los envases son más pequeños. 


\subsubsection{Reutiliza.}

Intenta alargar la vida de los objetos y en el caso de que el objeto no sirva para su función, intenta darle otros usos, antes de considerarlo como desecho. Estas son algunas ideas de cómo REUTILIZAR productos en casa: Utiliza las camisetas viejas como trapos de limpieza. Arregla los electrodomésticos, no los deseches a la primera. En lugar de desechar los envases, cajas y otros recipientes búscales otros usos o conviértelos en obras de arte. Compra baterías (pilas) recargables y no tires nunca pilas a la basura (el mercurio que contiene una batería puede contaminar un acuífero desde 600,000 hasta 2 millones de litros). No tires la ropa usada a la basura, dónala siempre hay alguien que la necesita. Utiliza todas las bolsas de plástico varias veces, o como bolsa de basura.

\subsubsection{Recicla.}

Reciclar en el hogar no es complicado, sólo hay que organizarse un poco. Lo más cómodo es poder disponer de cuatro cubos o recipientes diferentes. Papel y cartón: reciclando papel y cartón se puede ahorrar agua y energía, ya que para la fabricación de papel reciclado se gasta 100 veces menos agua y $70 \%$ menos de energía que produciéndolo a partir de madera. En la medida de lo posible, quita las grapas, clips y espirales antes de llevar el papel al contenedor. Puedes tirar al contenedor de papel, cajas de cartón, como cajas de huevos, de cereales, de galletas, de zapatos, etc. Además, sobres, periódicos, revistas, tiques de compra, cupones, facturas, entre otros.

No debes tirar al contenedor de papel, empaques tetrabrik, toallas o servilletas de papel, material sucio con alimento, como cajas de pizza, fotografías y radiografías, cajas revestidas con cera, metal u otro material que no sea papel o cartón. Plásticos: reciclando el plástico se reduce el consumo de petróleo ya que para cada kilogramo de plástico no reciclado se necesitan 2 kilogramos de petróleo crudo. En este contenedor puedes depositar envases 
de bebidas, tapones plásticos, bolsas de plástico y productos de plástico inyectado, como: cajillas, masetas, entre otros.

Tomar en consideración que el reciclando de metales como el acero o el aluminio, se puede llegar a ahorrar entre un 30 y un $70 \%$ de energía. En el contenedor de metales puedes depositar latas de bebidas, tapones de botellas y envases, y cualquier otro producto originado del metal. Materia orgánica y resto de desechos: El resto de desechos debes depositarlos en el camión de la basura para que sean llevados a los vertederos autorizados. Los restos de alimentos -incluyendo cáscaras, semillas, entre otros.

\section{Marco Metodológico}

La investigación se enmarca en el paradigma cualitativo interpretativo, buscando demostrar y comprender la realidad social de los fenómenos estudiados, su esencia, naturaleza y comportamiento; se destaca el valor e importancia de las ideas, sentimientos, motivaciones, vivencias e interrelaciones de los individuos en un contexto determinado, tratando de identificar la naturaleza de su realidad y su estructura dinámica en un escenario natural. De acuerdo con su tipo, se fundamenta en una metodología de Investigación Acción Participativa (IAP).

En este caso, se considera la IAP apoyada en una fase de campo, según Fals Borda, citado por Pérez (2017), esta investigación se relaciona más con una actividad de investigación propia de base popular sobre su realidad que con una acción receptiva de investigación realizada por élites ajenas a ellas (pág. 224). Resulta importante destacar ella, se ha convertido en una metodología de gran relevancia puesto que ha logrado complementar y a su vez multiplicar la partición protagónica, multiétnica y pluricultural de las comunidades y de organizaciones sociales.

El diseño de la investigación constituye la fase en la cual se identifica como se trabaja metodológicamente en la investigación, es decir, la estructura, 
como va a ser realizada, a la manera como la indagación es concebida a fin de obtener respuestas a las interrogantes. En este contexto, considerando el planteamiento anteriormente descrito, el diseño de esta investigación es de campo. En este sentido, Hernández y Col (2010), definen la investigación de campo como "Un proceso sistemático metódico y racional de recolección, comprobación, análisis e interpretación de datos, producto del contacto directo con un evento o proceso de la realidad en sus condiciones naturales" (pág. 39).

Esto se efectúa debido a que, para realizar la investigación y descripción actual del manejo de los desechos sólidos en el sector José Gregorio Hernández del municipio Pedraza, parroquia Ciudad Bolivia, la información se tomó directamente de la realidad, a través de un cuestionario dirigido a los habitantes de dicha comunidad, la cual fue el ámbito de estudio donde se obtuvo la información para dar cumplimiento a los objetivos de la investigación.

Para el presente estudio se considera como sujetos de estudios a sesenta y cinco (65) familias de las cuales se tomó el método de muestreo no probabilístico intencional y se seleccionaron como muestra veinte (20) familias de los cuales se tomaron tres (03) jefes de familia. Por tal razón, se consideraron sujetos con diferentes puntos de visita para que la información pudiera ser fidedigna; y así poder realizar un trabajo de presentación de hallazgos.

En este sentido, la información requerida para esta investigación fue recolectada a través de la técnica de la observación directa y la entrevista. La observación directa es definida por Chávez (2003), "Una forma de recoger información que generalmente se lleva a cabo en el contexto natural donde tienen lugar los acontecimientos" (pág. 48). Para esta investigación se realizó una observación incluida o participativa, puesto que el investigador participa en las tareas y actividades del grupo cuyas conductas quiere observar, aunque 
necesariamente participa en todas ellas, es decir, él observa desde dentro del grupo.

La finalidad del proceso de triangulación es obtener resultados sólidos que puedan orientar el trabajo de interpretación y estructuración de la información obtenida en la investigación para finalizar con la construcción del conocimiento. Cea (1999), cita a Denzin, el cual define a la triangulación en la investigación social como "La combinación de dos o más teorías, fuentes de datos, métodos de investigación, en el estudio de un fenómeno singular, en la triangulación se produce una comparación, un contraste desde diversas perspectivas" (pág. 43).

También se pueden triangular con fuentes de datos, su objetivo es provocar el intercambio de pareceres, comparar las diferentes perspectivas de los actores frente a un mismo fenómeno. Mientras que la información recolectada, debe ser organizada para codificar las respuestas de los encuestados, para luego realizar el análisis a través de la triangulación, finalmente se establecerán estrategias mediante actividades de acción. Al respecto Rojas (2010), toma en consideración los siguientes elementos:

El análisis de información tiene que ver directamente con la interpretación de lo que existe detrás de las realidades humanas, mediante las palabras, escritos, expresiones, se basa en analizar el cómo es la modalidad de su ejecución, desenvolvimiento y su determinación por aspectos como la que subyace a la realidad social (pág. 69).

La información se analiza después del proceso de recolección de los datos que no son estructurados pero que son muy variados y que luego el investigador le da estructura después de comprender con profundidad el contexto que lo rodea y relacionándolos con las teorías fundamentadas para posteriormente crear nuevas teorías. 


\section{Análisis de los Testimonios}

Tabla 1. Subcategoría: Recolección.

\begin{tabular}{|c|}
\hline Pregunta 4 \\
\hline $\begin{array}{l}\text { ¿Cuáles han sido las Alternativas de solución planteadas para la } \\
\text { recolección de los desechos sólidos? }\end{array}$ \\
\hline Sujeto 1: Opiniones \\
\hline $\begin{array}{l}\text { No se aplican alternativas relacionadas con la recolección de los desechos } \\
\text { sólidos. }\end{array}$ \\
\hline Sujeto 2: Opiniones \\
\hline $\begin{array}{l}\text { Debe ser tomada en cuenta el ordenamiento de la basura para incentivar } \\
\text { y sensibilizar a la población en relación al tema; y de esta manera forme } \\
\text { parte de las estrategias ecológicas que se desarrollaran en el sector. }\end{array}$ \\
\hline Sujeto 3: Opiniones \\
\hline $\begin{array}{l}\text { Con la adopción de un programa para la recolección de la basura se } \\
\text { plantearían normas que implican un convencimiento de la problemática } \\
\text { ambiental por parte de los involucrados produciendo un cambio en los } \\
\text { habitantes para habituarlos a los procesos de conservación ambientalista. }\end{array}$ \\
\hline Criterios: Opiniones \\
\hline $\begin{array}{l}\text { Se destaca en las respuestas dadas: Es deficiente la aplicación de } \\
\text { alternativas en relación con la recolección de los desechos sólidos como } \\
\text { elemento base de conservar el ambiente. Se evidencia la necesidad de } \\
\text { plantear alternativas en beneficio de un ambiente sano. }\end{array}$ \\
\hline
\end{tabular}

Fuente: Osorio (2016).

\subsection{Triangulación de la Entrevista.}

Los resultados de las entrevistas realizadas a los tres (3) jefes de familia que hacen vida activa en habitantes del sector José Gregorio Hernández. Se ejecuta observaciones de los testimonios, donde se recogen las evidencias que se desarrollaron en las actividades relacionadas con la aplicación del instrumento, los cuales constituyen la entrevista realizada de manera espontánea, en conversaciones abiertas con ellos.

Al respecto, Bizquerra citado por Rojas (2010), señalan la triangulación "Es una técnica para analizar los datos cualitativos, recogidos por diferentes 
técnicas, lo cual permite analizar una situación de diversos ángulos. "Es un control cruzado empleando diferentes fuentes, instrumentos o técnicas de recogida de datos" (pág. 4). De esta manera, la acción de reunión y cruce de la información pertinente al objeto de estudio en esencia constituye el corpus de resultados de la investigación. Por ello, la triangulación de la información es un acto que se realiza una vez que ha concluido el trabajo de recopilación de la información.

En la entrevista referida al conocimiento, de los testimonios dados por los sujetos de estudio se logró constatar que, en la institución la información que manejan acerca de la recolección de la basura es somera por lo que se debe promover un nuevo proceso ecológico apoyado en el trabajo colaborativo y contribuir con la preservación del ambiente.

Es importante difundir una conciencia ambientalista, porque la aplicación de programas conservacionista se debe involucrar a todos los ciudadanos de la localidad., así aumentar el trabajo ecológico, y preservar el ambiente. Por ello, es deficiente la aplicación de alternativas relacionadas con la recolección de los desechos sólidos como elemento base para la conservación del ambiente. Se evidencia la necesidad de plantear alternativas para establecer las responsabilidades y roles que le compete a cada ciudadano en beneficio de un ambiente sano.

Además, se desconoce de técnicas para la clasificación de la basura, lo cual se demanda de formas a usar para clasificar la basura en casa, mediante el trabajo en conjunto que instruya a toda a cada uno de los integrantes del hogar y la comunidad con relación a depositar la basura de acuerdo a su material. Asimismo, Es importante verificar las áreas más contaminadas por la basura mal colocada, así realizar una correcta recolección mediante la aplicación de estrategias ecológicas.

Por ello, Martínez (2014), expresa "La recolección y clasificación de la basura es una táctica para convertir los lugares que nos rodean en un entorno 
natural agradable donde se ponga en práctica actitudes y hábitos de cuidado y responsabilidad medio ambiental" (pág. 63). Sin embargo, se espera cultivar la conciencia ecológica y favorecer el ambiente, mediante acciones de clasificación, reutilización y reciclaje de desechos sólidos. De acuerdo con el tratamiento de los residuos sólidos los encuestados contestaron: Sería una buena idea, dado que en el municipio no existe ningún control sobre los mismos. Por último, dicen oportuno el establecimiento de relaciones con los entes gubernamentales y comunitarios.

\section{Planificación del Plan de Trabajo}

Tabla 2. Plan de Acción.

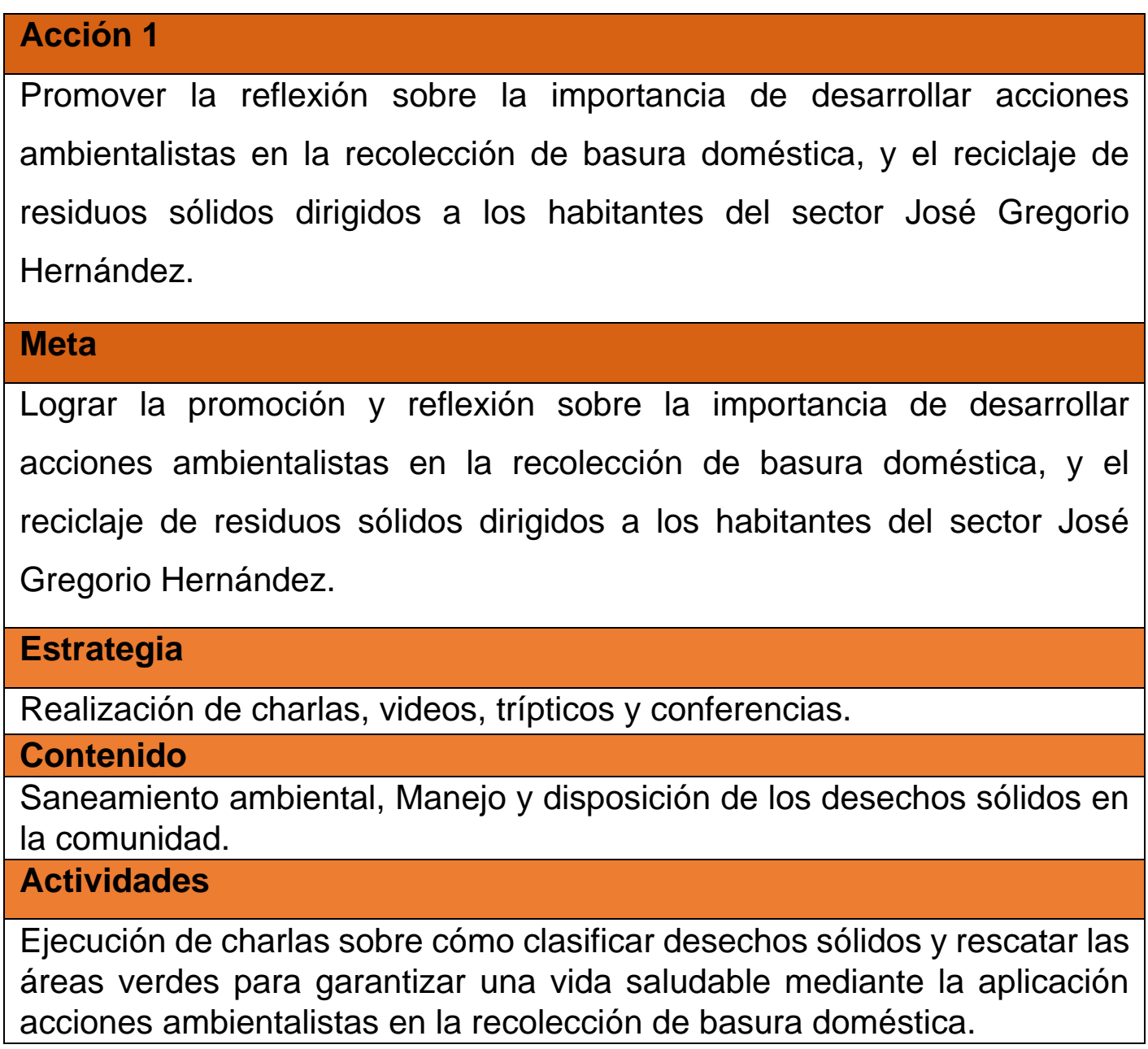


Ejecución de talleres: conservación del ambiente, trabajo colaborativo, Manejo y disposición de los desechos sólidos.

Elaboración de murales para exposición de las normas y acuerdos establecidos de rescate de áreas verdes a partir de acciones ambientalistas en la recolección de basura doméstica y el reciclaje.

\section{Recursos}

\section{Humano:}

Investigador, Habitantes.

Material:

Rotafolios, Televisor, Pizarra, Video Beam, Marcador acrílico.

\section{Tiempo}

Dos días en 03 sesiones.

Evaluación

Instrumento:

Registro fotográfico.

Criterios:

Discusiones grupales.

Facilitador

Eudis Ramón Osorio García

Fuente: Osorio (2016).

\section{Consideraciones Finales}

En la presente investigación se presentan las conclusiones y recomendaciones de los resultados obtenidos en función a los objetivos logrados. En cuanto a la promoción y reflexión sobre la importancia de desarrollar acciones ambientalistas en la recolección de basura doméstica, y el reciclaje de residuos sólidos dirigidos a los habitantes del sector José Gregorio Hernández; en relación con la jornada diaria en las áreas ambientales, donde la ausencia de estrategias e inclusión de procedimientos para compartir información y otras tareas ecológicas; durante el quehacer diario. 
En cuanto al diagnóstico obtenido de los habitantes del sector José Gregorio Hernández población estudiada se llegó a las siguientes consideraciones: Los encuestados están de acuerdo y dispuestos a desarrollar acciones ambientalistas en la recolección de basura doméstica, y el reciclaje de residuos sólidos de la comunidad, dado que, consideran pertinente trabajar y buscar alternativas para la integración comunitaria y la recolección de los residuos.

En lo que respecta a la factibilidad se concluye que es factible de ejecutar, puesto que cuenta con los organismos públicos y privados que le proporcionen la ayuda para la puesta en práctica del modelo autogestionario; asimismo, la población tiene la necesidad de conseguir solución a la ubicación de dichos desechos los cuales acarrean problemas, así mismo les servirá de guía para la optimización de la conservación y mantenimiento del ambiente y por ende de garantizar la calidad de vida de los habitantes.

Con la puesta en práctica de la propuesta referente a acciones ambientalistas en la recolección de la basura doméstica se consiguió llevar a la práctica actividades en función de las áreas afectadas por los residuos sólidos en áreas verdes donde cada uno de los habitantes, para lograr el reciclaje y manejo de los desechos, entre otra serie de actividades que serán necesarios para la continuación del programa.

Para la ejecución del plan se efectuaron convocatorias a los talleres y reuniones con el propósito de presentar el proyecto. Se puede mencionar que a través del desarrollo de acciones ambientalistas en la recolección de la basura doméstica, se logró el manejo adecuado de desechos sólidos en el Sector José Gregorio Hernández, como una acción que permite el mejoramiento del ambiente y salud de la comunidad.

\section{Recomendaciones}

De acuerdo con el plan desarrollado se presentan las siguientes 
recomendaciones: - Continuar la práctica de talleres relacionados con el plan, a fin de mantener informado a los habitantes de la comunidad. - Repetir trimestralmente los talleres de concientización ecológica relacionadas con la clasificación de la basura y el reciclaje. - Proponer programas de notificación a los habitantes de la comunidad con la finalidad que reflexionen acerca de la contaminación por desechos sólidos en el entorno inmediato.

\section{Referencias}

Blanco, A. (2012). Manejo de Desechos Sólidos por parte de los Habitantes de la Comunidad "El Samán de Urama" Estado Carabobo. Trabajo de grado para optar al título de Licenciado en Enfermería. Valencia, Venezuela: Universidad Central de Venezuela, Facultad de Medicina.

Caballero, Y. \& Col, R. (2006). Educación popular y educación ambiental: ¿un posicionamiento único?. La Habana: Editorial Caminos.

Cea, (1999). Proceso Investigativo. España: Editorial McGraw-Hill.

Chávez, M. (2003). Introducción a la investigación educativa. Caracas, Venezuela: Editorial Ars Graficas.

González, E. (2003). Educación para la ciudadanía ambiental. INTERCIENCIA. Vol. 28 N. 10.

Hernández, S. \& Col, O. (2010). Metodología de la investigación. México: Editorial. McGraw-Hill.

Martínez, R. (2007). Aspectos políticos de la educación ambiental. Revista INIE, Volumen 7, Número 3, págs. 1-25, UCR.

Martínez, R. (2012). Ensayo Crítico sobre Educación Ambiental. Revista Electrónica Diálogos Educativos, 12, 70-104. ISSN: 0718-1310. Recuperado de: https://issuu.com/umce/docs/dialogos24

Martínez, M. (2014). La Investigación Cualitativa Etnográfica en Educación. Trillas: México. 
Padilla, C. (2017). Análisis del Programa de Gestión de Residuos Sólidos en la Plaza de Mercado de Cerete - Córdoba. Universidad del Magdalena Estudiante Universidad de Manizales. Maestría en Desarrollo Sostenible y Medio Ambiente Santa Marta, Magdalena, Colombia.

Pérez, M. (2017). Investigación Acción Participativa (IAP) como Elemento de Fortalecimiento Educativo en la Escuela Bolivariana "EI Paramito", Estado Mérida. Revista Scientific, 2(4), 223-242. Recuperado de:

https://doi.org/10.29394/scientific.issn.2542-2987.2017.2.4.13.223-242

Rojas, S. (2010). La Investigación en Educación Ambiental como Herramienta Pedagógica. Editorial lberoamericana. México.

Temas Ambientales.com (2017). La Basura un Problema Ambiental. Recuperado de:

http://www.temasambientales.com/2017/03/basura.html 


\section{Eudis Ramón Osorio García}

e-mail: eudiseineidis@gmail.com

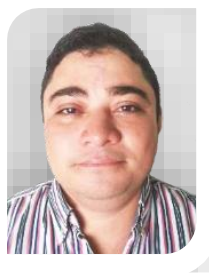

Nacido en Venezuela. Licenciado en Educación Integral, UNELLEZ, Barinas (2006). Cursante de la Maestría en Educación, Ambiente y Desarrollo, UPEL-IMPM. Desempeño laboral actual: Docente Coordinador de Educación Media y Diversificada. Experto en: Investigación. Manifiesto interés de formar parte de los árbitros de esta Revista. Línea de investigación del artículo: Educación Ambiente y Desarrollo, área Gestión Ambiental. 